\title{
Combinational Therpies and Drugs for Diabetes Mellitus Type I and Type II
}

\author{
Sivasankari S. ${ }^{1}$, Kumaravel S. ${ }^{2}$, Govardhanan K. ${ }^{3}$, Ganesh Kumar ${ }^{4}$ \\ ${ }^{I}$ Professor, Department of Microbiology, Meenakshi Medical College Hospital and Research Institute, Enathur, \\ Kanchipuram, Tamilnadu, India, ${ }^{2}$ Professor \& HOD, Department of Dermatology, Meenakshi Medical College \\ Hospital and Research Institute, Enathur, Kanchipuram, Tamilnadu, India, ${ }^{3}$ Professor, Department of General \\ Surgery, Meenakshi Medical College Hospital and Research Institute, Enathur, Kanchipuram, Tamilnadu, India, \\ ${ }^{4}$ Assistant Professor, Department of Pharmacology, Meenakshi Medical College Hospital and Research Institute, \\ Enathur, Kanchipuram, Tamilnadu, India
}

\begin{abstract}
Diabetes mellitus is a metabolic disorder causes changes in the blood glucose level either it can be elevated or lowered. These are classified into two type insulin dependent and insulin non-dependent. The basic treatment provided for diabetic patients are insulin therapy it has been clinically proven but this is not the only solution to cure diabetes. Metformin is widely used medicine for patients. Glucose lowering therapy is applicable for patients with severe hyperglycemia, hepatic and renal related diseases. The therapeutic applications such as The therapeutic medicines exenatide and to induce the insulin secretion and also liraglutide helps people with insulin. Other therapeutic compounds heals the defects of beta-cells secretion are compensated with the use of stitagliptin to inhibit the dipeptidyl peptidase-4 (DPP-4) enzyme, and increases the the function of Islets cells. This article further discusses about the combinational therapies available for the diabetic patients.
\end{abstract}

Keywords: Diabetes, therapies, drugs, mellitus.

\section{Introduction}

Diabetes mellitus is considered as life-threatening metabolic disorder worldwide. In recent survey around 300 million people got affected with this disease with the diagnosed rate of $8 \%$ and $50 \%$ undiagnosed ${ }^{[1]}$. The maximum people with middle income are found to be diabetic related with stress, unhealthy diet with the age group of 45-55 in the population.In the estimation of recent survey by ICMR collaboration with china predicts in the survey an estimation of 99 million people with the

\section{Corresponding Author:}

\section{Sivasankari S.}

Professor, Department of Microbiology, Meenakshi

Medical College Hospital and Research Institute, Enathur, Kanchipuram, Tamilnadu, India

e-mail: sivasankaris@mmchri.ac.in case history and the future predicted survey says that in upcoming years 6 million people are tend to get affected. There are few incidence that makes people with diabetic are prone to cardiac related disease in the east Asian countries. There are chances to get diabetic in people with obesity. There are the major health complications in diabetic patients ${ }^{[2]}$. The drug metformin is utilized as the combinational therapy medicine for type 2 diabetic patients. It was available in Europe and U.S. and approved in the year 1995. The usage of metformin has adverse effects for the patients with increased level of serum creatinine. It should be properly prescribed with $\mathrm{mg}$ level in elder patients around 80 yrs of age because of the complications in renal functioning [3]. Attaining the targeted glycaemic level is of utmost importance the initial stage in use of combinational medicines leads to reduction in HbAlc. Combinational therapy for types 2 diabetic is more risky than type 1 because type has more metabolic defects. Hypoglycemic patients cells can have an impactful memory of vascular cells of the 
target organs creating a metabolic memory leads to complications in future.Diabetic people those who are with poor control leads to develop risk factors ${ }^{[4]}$.

Preclinical studies in recent establishment of medicines for diabetes: FDA has approved many drugs of combinational therapy for diabetic patients also to avoid side effects which leads to immunosuppression in patients. The ultimate standardization approach to achieve approval can be developed. Type 1 diabetic patients approval of medicines requires a panel of members with animal models to develop medicines for FDA approval. To ensure even formulations of medicines it is compared with standards and compared with operating procedures it is presented and multiple data has been stored. Such consortium can consist diverse laboratories developing the preclinical models in standardizing the procedures to get the approval from Diabetes combination therapies assessment group. This formation will allow two or three labs work to test the combinational medicines. The protocols from all laboratories will put to standardization so that the therapeutic medicines turn as a GMP material known as good manufacturing practise. The ultimate goal of this initiatives are to provide quality preclinicaldatafor the novel combinational medicines therapies for type 1 diabetic medicines ${ }^{[5]}$.

\section{Type I Diabetes mellitus Combinational Medicines:}

Rapamycin: It is an inhibitor of non-calcineurin used in the prevention of graft rejection after the allogenic transplant. Additionally administering the rapamycin drug prevents the type 1 diabetes in mice. This drug rapamycin drug for humans provided for juvenile for beta cell replacement diabetes research foundation center. The research studies revealed that recipients of renal transplant t-cell depletion by inducing CAMPTH-1H. The clinical protocol in using rapamycin therapy is provided to type 1 diabetic patients before transplantation of islets to reach therapeutic plasma level before infusion of islets that follows rapamycin mono therapy. Rapamycin has an effect on human nTreg function, which supports its clinical immuno suppressant agents for tolerance.

\section{Stem Cell Therapy $\beta$-Cell Immunomodulation:} BETA-cells for therapy is a major challenge. Limitations of using stem cells represents ultimate potential of stem cells to regenerate the cells and immunomodulation potential. The capacity to renew to turn from differentiate into specialized cells can be a useful for glucose supply and insulin producing cells for transplantation. The mesenchyma cells and hematopoetic cells can aid to retrieve the beta cell destructors and facilitates the regeneration of cells. Hence the cells with these potency can be utilized in beta-cell therapies to reverse hyperglycemic in type 1 diabetic patients. These Stem cells can be produced from various sources obtained to test their potential to restore homeostasis or to enhance the survival in transplant patients. It includes pluripotent stem cells, embryonic stem cellsand bone marrowmesenchymal cells and adipose tissue-derived cells and the cells that secretes in the ductal epithelium Beta-cell progenitors are found ${ }^{[6]}$.

Peptide Hormone Therapies: Peptide hormone mimics the cells. The peptide (GLP-1) Glucagon-like peptide are widely being used to treat the diabetes and obesity. Pre-clinical data deposited that strongly suggests the benefits of many hormonal peptides, which develops the potential type 1 diabetes treatment. Including the potential regulation of glucose and secretion of insulin, beta cell preservation and increases the insulin sensitivity. The pre-clinical study exposes the GLP1 therapeutic utilization of insulin in type- 1 diabetic patients. It demonstrates the reduction of postprandial glucose excursions, production of decreased glucagon and the delaying of empty the gastric in patients insulin uptake and combination with GLP-1R as compared with insulin therapy. The study demonstrates the controlled glycaemic level in combinational therapy with anti-IL2 antibody ${ }^{[7]}$.

\section{Therapeutic Drugs for Type II Diabetes mellitus:}

Type II a-glucosidae inhibitors: Acarbose, miglitol and the voglibose are the currently available medicines for type 2 diabetic patients. These medicines are currently available for anti-diabetic property it possess for the mode of action. Mainly the acarbose has been utilized for more than 10 years in the treatment of diabetic mellitus. These inhibitors take role in reducing the triglycerides but improving their effect on low density and high density lipoprotein levels are inconsistent. This inhibitor sometimes rarely induce the hypoglycemiathese do not stimulate the insulin level and do not disturb the weight of patients. Acarbose inhibitor has reduced the risk of cardiac related disease shows progression in patients with glucose intolerance ${ }^{[8]}$. 
Gut Microbiota: Human gut consist beneficial bacterium, identified as gut microbiota. These microorganisms consists essential functions gut microbiota serves several essential functions in vitamin production such as vitamin $\mathrm{k}$, folic acids to enhance energy production. Research studies have reported that the colonizing bacteria contact happens in birth canal. In this therapy the microbiomes have found in amniotic cavitiy, amniotic fluid and in umbilical cord.In infants the microbiota composed of materials are completely different from adult people. After three years of birth the microbiota has developed to the maturation stage which is essential throughout the adult stage. The major diseases are prevented by the developments in human body. Studies revealed that animal models and in adults had demonstrates that microbiota improper function can lead to the development of obesity in type 2 diabetic patients. There are chances of fluctuation in the energy level of healthy individuals these inhibits the insulin resistant and and develops dyslipidemia are developed by low anti-bacterial activity ${ }^{[9]}$.

Thiazolidinediones: These Troglitazone has been found in the year 1997 in the type 2 diabetes milletus. These are removed from the production companies because it causes liver toxicity. With the use of medicines patients gained weight after taking troglitazone medicine. This medicine sticks with the peroxisome activator proliferation and gamma nuclear receptors affects the gene regulation. This alteration transcription of gene takes place in adipocytes and the primarily concerned genes participate in regulation of fat metabolism. This reduction in fatty acids eventually regulates the production of insulin. This FFA develops the beta cell function by reducing the lipotoxicity. The toxicity leads to cell death in beta cells ${ }^{[10]}$.

Pioglitazone: This drug is used as a mono-therapeutic agent combined with metformin (increases antihyperglycemia). These compounds such as meglitinide works well in diabetic patients with metabolic disorder associated with type 2 diabetic patients. Low dosage is recommended per day. It shows a relevant mode of action and adverse effects in lever toxicity when compared to rosiglitazone. It can also interact with another medicine that are functioned by enzyme p45 and change the level of serum. Contraceptive drugs used in higher dosage in diabetic female who does not want to get conceived ${ }^{[11]}$.

Sulfonylureas: The first and second generation nature of this compound binds with the receptor of sulfonylurea present in the pancreatic beta cells surface and it ultimately leads to the increased range of secreting insulin. These cause changes in potassium channel and depolarization in the membrane of the cell. These calcium channel pave way to an influx in calcium and increases insulin secretion in pancreas. The first generation medicines are with low potency compounds include (diabinase, dymelor, to linase). The second generation medicines easily penetrate through the cell membranes ${ }^{[12]}$.

\section{Sodium Glucose Cotransporter 2 Inhibitors:}

These inhibitor targets the organ kidney to develop the glucose in excreta from urine and eventually decreases hyperglycemic condition. In normal condition, organ reabsorbs the glucose filtered. Hence no glucose is excrete from the urine samples. Glucose re absorption happens at the proximal tube, by the help of glucose protein SGLT2. The expression of this protein seem to be increases in type 2 diabetic patients. Re-absorption capacity is increased by inhibiting the protein for reabsorption of glucose decreases the hyperglycemic condition. This inhibitors can be utilized in different combinations, different types of agents aids in lowering the glucose ${ }^{[13]}$.

Hybrid chimeric peptides: The combinations of two peptides are injected to the patients has developed with the molecules hybrid are two peptide molecules and the amino acids linked the peptide molecules to form a single compound. For instance glugacon peptide has been mixed with the gastrin eventually improves the control glycaemic condition in patients and also controls the beta cell mass. It is also linkswith glucagon improves the energy level and leads to weight reduction. Possibility to arrange chimeric peptides with good properties to deliver the desirable effects through the receptors of targeted cells. The acute reaction, immunogenecity and physio chemical challenges are considered in the development of drugs ${ }^{[14]}$.

Patho-Therapeutic processes: The action of anti-diabetic medicine is pharmacologically inactive sometimes. In the beginning stage, pharmacologists focus in the sugar level in blood and intake of food and digestive compounds related to glucose. Development of novel drugs for the type 2 diabetes milletus should be diverse and helpful without any complications. Therapeutic medicines are related with coronary diseases and obesity that should be avoided while designing a drug. Experimental studies should develop and enhance 
the mode of action of drugs. Accordingly, we should not rely on one or two therapeutic pathways ${ }^{[15]}$. Generally these medicines are not toxic when compared to high risk synthetic drugs.

Exenatide: This drug mimics the incretin protein which has glucose regulating molecules having glucoregulatory activities related to hormonal peptide glucagon. The activity includes the blood glucosedependency enhances the secretion of insulin. The drug enhances the glucose level for secretion of insulin mediates the binding of exenatide with glucagon with pancreatic cells. The glucagon peptide improves the cell function by using the drug in exenatide by increasing the gene expression involved in secretion of insulin and the augment the beta cell mass. This peptide controls the intake of food and helps in weight loss increases insulin effect in body ${ }^{[16]}$.

\section{Conclusion}

These therapeutic drugs are available in recent years considering uncontrollable diabetic cases in India and all over the world. The developed countries life style and lethargic manner in junk food intake are also the reasons for developing diabetic mellitus metabolic disorder. The therapeutic drugs mainly helps to reduce the weight and improves the metabolic rate in diabetic patients. This should be taken into consideration by people to change their idea of having irresponsible changes in life style. The use of combinational therapeutic drugs will eventually help people with type 1 and type 2 diabetic patients for the well being of highest population.

\section{Ethical Clearance: Nil}

Source of Funding: Meenakshi Academy of Higher Education and Research, Chennai, India

\section{Conflict of Interest: Nil}

\section{References}

1. Otto-Buczkowska E, Jainta N. Pharmacological treatment in diabetes mellitus type 1-insulin and what else?. International journal of endocrinology and metabolism. 2018 Jan;16(1).

2. Tiwari P. Recent trends in therapeutic approaches for diabetes management: a comprehensive update. Journal of diabetes research. 2015 Jan 1;2015.

3. Bailey T. Options for combination therapy in type 2 diabetes: comparison of the ADA/EASD position statement and AACE/ACE algorithm. The American journal of medicine. 2013 Sep 1;126(9):S10-20.

4. Cahn A, Cefalu WT. Clinical considerations for use of initial combination therapy in type 2 diabetes.

Diabetes Care. 2016 Aug 1;39(Supplement 2):S137-45.

5. Matthews JB, Staeva TP, Bernstein PL, Peakman M, Von Herrath M, ITN-JDRF Type 1 Diabetes Combination Therapy Assessment Group. Developing combination immunotherapies for type 1 diabetes: recommendations from the ITN-JDRF Type 1 Diabetes Combination Therapy Assessment Group. Clinical \& Experimental Immunology. 2010 May;160(2):176-84.

6. Chhabra P, Brayman KL. Stem cell therapy to cure type 1 diabetes: from hype to hope. Stem cells translational medicine. 2013 May;2(5):328-36.

7. Haslacher H, Fallmann H, Waldhäusl C, Hartmann E, Wagner OF, Waldhäusl W. Type 1 diabetes care: Improvement by standardization in a diabetes rehabilitation clinic. An observational report. PloS one. 2018 Mar 12;13(3):e0194135.

8. Pathak V, Pathak NM, O’Neill CL, Guduric-Fuchs J, Medina RJ. Therapies for type 1 diabetes: current scenario and future perspectives. Clinical Medicine Insights: Endocrinology and Diabetes. 2019 May;12:1179551419844521.

9. Vezza T, Abad-Jiménez Z, Marti-Cabrera M, Rocha M, Víctor VM. Microbiota-Mitochondria Inter-Talk: A Potential Therapeutic Strategy in Obesity and Type 2 Diabetes. Antioxidants. 2020 Sep;9(9):848.

10. Sheehan MT. Current therapeutic options in type 2 diabetes mellitus: a practical approach. Clinical medicine \& research. 2003 Jul 1;1(3):189-200.

11. Pontarolo R, Sanches AC, Wiens A, Perlin CM, Tonin FS, Borba HH, Lenzi L, da Silva Penteado ST. Pharmacological Treatments for Type 2 Diabetes. Treatment of Type 2 Diabetes. 2015 Apr 1:147.

12. Brunetti L, Kalabalik J. Management of type-2 diabetes mellitus in adults: focus on individualizing non-insulin therapies. Pharmacy and Therapeutics. 2012 Dec;37(12):687.

13. Thrasher J. Pharmacologic management of type 2 diabetes mellitus: available therapies. The American journal of cardiology. $2017 \mathrm{Jul}$ 1;120(1):S4-16. 
14. Bailey CJ, Day C. Treatment of type 2 diabetes: future approaches. British medical bulletin. 2018 Apr 18;126(1):123-37.

15. Lu DY, Che JY, Yarla NS, Zhu H, Lu TR, Xu B, Putta S. Type 2 diabetes study, introduction and perspective. The Open Diabetes Journal. 2018 Jul $31 ; 8(1)$.
16. Buse JB, Henry RR, Han J, Kim DD, Fineman MS, Baron AD. Effects of exenatide (exendin-4) on glycemic control over 30 weeks in sulfonylureatreated patients with type 2 diabetes. Diabetes care. 2004 Nov 1;27(11):2628-35. 